\title{
mir-1-mediated paracrine effect of cancer-associated fibroblasts on lung cancer cell proliferation and chemoresistance
}

\author{
JIANMIN LI ${ }^{1,2}$, JING GUAN $^{3}$, XIAOPING LONG ${ }^{4}$, YANG WANG ${ }^{5}$ and XUDONG XIANG ${ }^{6}$ \\ ${ }^{1}$ Department of Respiratory Medicine, The Second Xiangya Hospital of Central South University, Changsha, Hunan 410011; \\ ${ }^{2}$ Department of Respiratory Medicine, Hunan Provincal People's Hospital, Changsha; ${ }^{3}$ Department of Emergency Medicine, \\ The First Hospital of Changsha, Changsha, Hunan 410005; ${ }^{4}$ Department of Respiratory Medicine, The First Affiliated Hospital \\ of the University of South China, Hengyang, Hunan 421000; Departments of ${ }^{5}$ Pathology and ${ }^{6}$ Emergency Medicine, \\ The Second Xiangya Hospital of Central South University, Changsha, Hunan 410011, P.R. China
}

Received December 5, 2015; Accepted January 8, 2016

DOI: $10.3892 /$ or.2016.4714

\begin{abstract}
Lung cancer is the leading cause of cancer-related mortality in humans worldwide. Moreover, the overall 5-year survival rate is only $15 \%$. Pathologically almost $80 \%$ of all lung cancer cases are non-small cell lung cancer (NSCLC). Cancer-associated fibroblasts (CAFs) have been found to exist in a large number of NSCLCs. CAFs have been proven to promote tumor progression, metastasis and resistance to therapy through paracrine effects in most solid tumors. In the present study, firstly we isolated CAFs from patient tissues and demonstrated that they promoted cell proliferation and chemoresistance to cisplatin in the lung cancer cell lines A549 and 95D in a paracrine manner. Secondly, using ELISA and quantative PCR, we found that a higher amount of stromal cellderived factor 1 (SDF-1) existed in the CAFs rather than that observed in the normal fibroblasts (NFs). Thirdly, we detected that SDF-1 facilitated lung cancer cell proliferation and drug resistance via the CXCR4-mediated signaling pathway which involved NF- $\mathrm{KB}$ and $\mathrm{Bcl}-\mathrm{xL}$. Moreover, we also confirmed that the expression level of SDF-1 in the CAFs was negatively regulated by microRNA mir-1 through microRNA overexpression and quantitative PCR. Overall, our data provide one explanation for the effects of CAFs on lung cancer cells. Meanwhile, our results also suggest CAFs as a potential therapeutic target in tumor treatment.
\end{abstract}

\section{Introduction}

Lung cancer is reported to be the leading cause of cancer-related mortality in both men and women worldwide $(1,2)$. Moreover,

Correspondence to: Dr Xudong Xiang, Department of Emergency Medicine, The Second Xiangya Hospital of Central South University, 139 Middle Renmin Road, Changsha, Hunan 410011, P.R. China E-mail: xudongx_99@hotmail.com

Key words: lung cancer, mir-1, cancer-associated fibroblasts, SDF-1, CXCR4, cell proliferation, chemoresistance, NF- $\kappa B, B c 1-x L$ the reported overall 5-year survival rate is only $15 \%$ partially due to the low efficacy of current treatment techniques (1). Thus, novel treatments are urgently needed for this type of cancer. Pathologically almost $80 \%$ of all lung cancer cases are non-small cell lung cancer (NSCLC). Cancer-associated fibroblasts (CAFs) have been found to exist in a large number of NSCLCs (3). With the development of its research, CAFs have been proven to promote tumor progression, metastasis and resistance to therapy through paracrine effects in most solid tumors $(4,5)$. Soluble factors secreted from CAFs include TGF- $\beta$ family members, EGF family members, chemokines and many other molecules. Among all these factors, stromal cell-derived factor 1 (SDF-1), which is expressed at elevated level in CAFs, was discovered to perform an important role in tumor cell survival, metastasis and chemoresistance through activation of its receptor, CXCR4 (4). However, how SDF-1 functions during the interaction between CAFs and lung cancer cells is not yet completely clear.

Chemokine receptors are one type of seven transmembrane G-protein-coupled receptors. They are classified into four different groups as $\mathrm{CXC}, \mathrm{CX} 3 \mathrm{C}, \mathrm{CC}$ and $\mathrm{XC}$ according to the chemokines they primarily interact with for signaling (6). CXCR4, one member of the chemokine receptor family, has been found to be overexpressed in more than 23 different types of cancers including lung, prostate and breast cancer as well as leukemia $(6,7)$. It exerts its function in tumor cell survival, metastasis and therapeutic resistance mainly through the PI3K/AKT/NF- $\kappa \mathrm{B}$ axis as well as the ERK1/2/NF- $\kappa \mathrm{B}$ axis after interaction with its ligand SDF-1 (8). Meanwhile, activation of $\mathrm{NF}-\kappa \mathrm{B}$ has been linked to tumor cell survival and cancer therapy resistance partially through regulating the expression level of $\mathrm{Bcl}-\mathrm{xL}$, one member of the anti-apoptotic genes (9). However, whether NF- $\mathrm{BB}$ and $\mathrm{Bcl}-\mathrm{xL}$ are involved in the interaction between CAFs and lung cancer cells is not quite certain.

Although SDF-1 as well as many other soluble factors are highly expressed in CAFs, the molecular mechanisms controlling their expression levels are unclear. MicroRNAs, since firstly discovered in 1993, have been reported to play important roles in many aspects of cell biology, including cell proliferation, apoptosis, cell cycle and carcinogenesis (10). 
Numerous studies have demonstrated that the expression of microRNAs in tumors are either upregulated or downregulated compared with their expression level in normal tissues (11). Downregulation of microRNAs has been discovered to be related with tumor suppression. As one of the tumor-suppressing microRNAs, mir-1 was found to be consistently expressed at a very low level in many tumors including lung cancer (12), which was consistent with our data shown in Fig. 2C. Moreover, mir-1 was found to directly regulate the expression of SDF-1 and CXCR4 in thyroid cancer (13). However, whether mir-1 targets SDF-1 in CAFs and affects its role in the interaction between CAFs and lung cancer cells has not yet been reported. Therefore, in the present study, by employing lung cancer cell lines A549 and 95D and isolation of primary CAFs, we investigated the involvement of SDF-1 in the interaction between CAFs and lung cancer cell lines and demonstrated that SDF-1 was able to activate $\mathrm{NF}-\kappa \mathrm{B}$ and Bcl-xL by interacting with CXCR4 in the A549 and 95D cells. Furthermore, in our experiments, mir-1 was confirmed to negatively regulate the expression of SDF-1. In conclusion, we discovered that by secretion of SDF-1, which was regulated by mir-1, CAFs were able to enhance cell proliferation and the drug resistance to cisplatin in the A549 and 95D cells. These results also suggest CAFs as a potential therapeutic target in tumor treatment.

\section{Materials and methods}

Reagents and antibodies. Dulbecco's modified Eagle's medium (DMEM):nutrient mixture F-12 (DMEM/F-12) and fetal bovine serum (FBS) were purchased from GE Healthcare HyClone (Logan, UT, USA). Rabbit anti-human fibroblast activation protein (FAP), mouse anti-human $\alpha$-smooth muscle actin ( $\alpha$-SMA), mouse anti-human CXCR4, mouse anti-human $\mathrm{NF}-\kappa \mathrm{B}$ and rabbit anti-human $\beta$-actin antibodies were provided by Cell Signaling Technology (Danvers, MA, USA). Mouse anti-human $\alpha$-SMA and rabbit anti-human Bcl-xL antibodies were obtained from Santa Cruz Biotechnology (Dallas, TX, USA). Goat anti-mouse IgG/HRP, goat anti-rabbit IgG/HRP, FITC-labeled goat anti-mouse IgG and TRITC-labeled goat anti-rabbit IgG were purchased from KPL, Inc. (Gaithersburg, MD, USA). All primers for mir-1, SDF- $1 \alpha, \alpha$-SMA, FAP, U6 and GAPDH as well as the sequence of CXCR4 siRNA and mir-1 mimics were synthesized by GenePharma (Shanghai, China). Human CXCR4 plasmid was constructed by Yrbio (Changsha, China).

Primary lung cancer CAF and normal fibroblast $(N F)$ cultures. Lung cancer and normal lung tissues were obtained from patients with lung cancer after obtaining informed written contents. Normal lung tissues were collected from the same patients at locations at least $5 \mathrm{~cm}$ away from the tumor sites. Both types of tissues were kept in DMEM/F12 supplemented with penicillin $(100 \mathrm{U} / \mathrm{ml})$ and streptomycin $(50 \mu \mathrm{g} /$ $\mathrm{ml})$. Primary cell isolation was performed within $2 \mathrm{~h}$ after excision. Briefly, lung cancer and normal lung tissues were cut into small pieces with a size of $\sim 1 \mathrm{~mm}^{3}$ and planted in cell culture flasks. DMEM/F12 supplemented with $10 \% \mathrm{FBS}$, penicillin $(100 \mathrm{U} / \mathrm{ml})$ and streptomycin $(50 \mu \mathrm{g} / \mathrm{ml})$ were added to the flasks. Seventy-two hours later, the cells outgrew from the explant culture and medium was changed gently. Then, afterwards, the medium was changed twice a week. Subculture was performed when cells reached $80 \%$ confluency at the split ratio of $1: 3$.

Immunofluorescence staining. CAFs and NFs at passages 2-4 were seeded on coverslips in a 24-well plate on the day before staining. On the next day, the cells were fixed with $4 \%$ paraformaldehyde for $30 \mathrm{~min}$ at room temperature. After permeabilization with $0.3 \%$ Triton X-100 for $5 \mathrm{~min}$, the cells were incubated with primary antibodies to FAP and $\alpha$-SMA overnight at $4^{\circ} \mathrm{C}$. Then, on the second day, FITC and TRITClabeled secondary antibodies were added after washing for 3 times with 1X PBS and kept for $2 \mathrm{~h}$ at room temperature. Finally, the coverslips were mounted on microslides and examinated under an inverted fluorescence microscope. Images were captured under a magnification of x200.

MTT assay. Ten microliters of MTT $(5 \mathrm{mg} / \mathrm{ml})$ were added into 96-well plates with cells at 5,000 cells/well and incubated for $4 \mathrm{~h}$ in cell incubators at $37^{\circ} \mathrm{C}$ in the absence of light. Then, culture medium was gently damped and dimethyl sulfoxide (DMSO) was added to stop the reaction. Optical densities (ODs) were measured with a microplate reader at $490 \mathrm{~nm}$.

Enzyme-linked immunosorbent assay (ELISA). The secretion of SDF-1 from CAFs and NFs into culture medium was measure by SDF-1 $\alpha$ (human) ELISA kit (Abnova, Taipei, Taiwan). CAFs and NFs were maintained in T-25 culture flasks. When cells reached confluency, the medium was replenished with $5 \mathrm{ml}$ fresh medium. Twenty-four hours later, the culture supernatant was collected and kept at $-80^{\circ} \mathrm{C}$ before measurement. All steps were carried out according to the manufacturer's instructions.

CXCR4 overexpression and silencing. CXCR4 upregulation or downregulation in the A549 and 95D cells were carried out with CXCR4 plasmids or CXCR4 siRNA transfection with Lipofectamine 2000 in a 6-well plate, respectively. The sequences of CXCR4 siRNA were:5'-GGGACUAUGACUCCA UGAATTUUCAUGGAGUCAUAGUCCCTT-3'. All steps were carried out according to the manufacturer's instructions. Western blotting was used to evaluate the transfection efficacy $24 \mathrm{~h}$ after transfection.

Assessment of cell proliferation. The cell proliferation rate of the A549 and 95D cells after CXCR4 overexpression and silencing was measured with MTT assay. Cells were divided into 3 groups: cells only, cells + CXCR4 (CXCR4-overexpressing cells) and cells + CXCR4 siRNA (CXCR4-silenced cells). Twenty-four hours after transfection, the cells were seeded into a 96-well plate, and MTT assay was carried out 24, 48 and $72 \mathrm{~h}$ after cells were seeded.

The cell proliferation rate of the A549 and 95D cells after incubation with CAFs or addition of the CAF supernatant was assessed with the MTT assay. In the present study, AMD3100, one type of CXCR4 inhibitor and recombinant human chemokine SDF-1 were used to verify their effect on the proliferation rate of the A549 and 95D cells. The cells in the present study were divided into groups as follows: cells only, cells + CAF 
supernatant, cells $+10 \mathrm{ng} / \mathrm{ml} \mathrm{SDF-1,} \mathrm{cells} \mathrm{+} \mathrm{CAFs} \mathrm{co-culture,}$ and cells + CAFs co-culture + $10 \mu \mathrm{g} / \mathrm{ml}$ AMD3100. A549 or $95 \mathrm{D}$ cells were seeded into a $96-$ well plate $24 \mathrm{~h}$ before co-culturing with CAFs or addition of SDF-1, AMD3100 or CAF supernatant. Afterwards, MTT assay was carried out 24, 48 and $72 \mathrm{~h}$, later.

Assessment of the drug resistance to cisplatin. Drug resistance of the A549 and 95D cells after CXCR4 overexpression and silencing was measured using the MTT assay. The cells were divided into 3 groups: cells only, cells + CXCR4 (CXCR4-overexpressing cells) and cells + CXCR4 siRNA (CXCR4-silenced cells). Twenty-four hours after transfection, the cells were seeded into a 96-well plate, and cisplatin was added on the next day. Concentrations of cisplatin applied in the present study were $0,0.6,1.2,2.4,3.6$ and $7.2 \mu \mathrm{g} / \mathrm{ml}$. MTT assay was carried out $24 \mathrm{~h}$ after cisplatin was added. Cell viability at the concentration of $0 \mu \mathrm{g} / \mathrm{ml}$ was set as $100 \%$; cell viability at all other concentrations was calculated accordingly.

The drug resistance of the A549 and 95D cells after incubation with the CAFs or addition of the CAF supernatant was tested using the MTT assay. In the present study, AMD3100, one type of CXCR4 inhibitor and recombinant human chemokine SDF-1 were used to verify their effect on the drug resistance of the A549 and 95D cells. The cells in the present study were divided into groups as follows: cells only, cells + CAF supernatant, cells $+10 \mathrm{ng} / \mathrm{ml} \mathrm{SDF-1}$, cells + CAFs co-culture, and cells + CAFs co-culture $+10 \mu \mathrm{g} / \mathrm{ml}$ AMD3100. A549 or 95D cells were seeded into a 96 -well plate $24 \mathrm{~h}$ before co-culturing with CAFs or the addition of SDF-1, AMD3100 or CAF supernatant. Cisplatin was added also on the next day after cells were seeded. Concentrations of cisplatin applied in the present study were $0,0.6,1.2,2.4,3.6$ and $7.2 \mu \mathrm{g} / \mathrm{ml}$. MTT assay was carried out $24 \mathrm{~h}$ after cisplatin was added. Cell viability at the concentration of $0 \mu \mathrm{g} / \mathrm{ml}$ was set as $100 \%$; cell viability at all other concentrations was calculated, accordingly.

MicroRNA mir-1 overexpression. The expression level of mir-1 in CAFs was elevated by transient mir-1 mimic transfection with Lipofectamine 2000 in a 6-well plate. The duplex sequences of mir-1 mimics were: sense, 5'-ACAUACUUCUU UACAUUCCATT-3' and antisense, 5'-UGGAAUGUAAAGA AGUAUGUAU-3'. All steps were carried out according to the manufacturer's instructions. Quantitative PCR was utilized to evaluate the transfection efficacy 24 and $48 \mathrm{~h}$ after transfection.

Quantative PCR. Total RNA was extracted from the cells using TRIzol reagent and used for cDNA synthesis. mir-1 reverse transcription was conducted with a microRNA-specific primer using the miScript Reverse Transcription kit (Qiagen, Hilden, Germany). For mRNA reverse transcription, total RNA was reverse transcribed with the SuperScript Reverse Transcription kit (Thermo Fisher, Waltham, MA, USA). Quantitative real-time PCR was performed using SYBRGreen Master Mix (Bio-Rad, Hercules, CA, USA). The following primers were used: mir-1-forward, 5'-CTG TCACTCGAGCTGCTGGAATG-3' and mir-1-reverse, 5'-ACCGTGTCGTGGAGTCGGCAATT-3'; SDF-1 $\alpha$-forward, 5'-CCAAACTGTGCCCTTCAGAT-3' and SDF-1 $\alpha$-reverse,
5'-CTTTAGCTTCGGGTCAATGC-3'; $\alpha$-SMA-forward, 5'-AGCTACCCGCCCAGAAACTA-3' and $\alpha$-SMA-reverse, 5'-ATGATGCCGTGCTCGATAGG-3'; FAP-forward, 5'-TGT GCATTGTCTTACGCCCT-3' and FAP-reverse, 5'-GAG TATCTCCAAAGCATGGTTCTA-3'; GAPDH-forward, 5'-CCAGGTGGTCTCCTCTGA-3 and GAPDH-reverse, 5'-GCTGTAGCCAAATCGTTGT-3'; U6-forward, 5'-CTC GCTTCGGCAGCACA-3' and U6-reverse, 5'-AACGCT TCACGAATTTGCGT-3'. The mRNA expression values were normalized to GAPDH. The microRNA expression values were normalized to U6. Relative expression levels of microRNA or mRNA were analyzed using the Bio-Rad C1000 Thermal Cycler.

Western blotting. Whole-cell protein was extracted with protein lysis solution [50 mM Tris- $\mathrm{HCl}(\mathrm{pH} 7.5), 150 \mathrm{mM}$ $\mathrm{NaCl}, 1 \% \mathrm{SDS}, 0.5 \%$ sodiumdeoxycholate and $0.5 \%$ Triton X-100] on ice. Protease inhibitors were added into the cellular lysate. The protein concentration was measured using a bicinchoninic acid assay. Western blot analysis was performed as previously described (14) using the following antibodies: mouse anti-human CXCR4, mouse anti-human $\mathrm{NF}-\kappa \mathrm{B}$, rabbit anti-human $\mathrm{Bcl}-\mathrm{xL}$ and rabbit anti-human $\beta$-actin antibodies. Briefly, equal amounts of protein (50 ng) were separated by SDS-PAGE and transferred to polyvinylidene difluoride (PVDF) membranes. HRP-conjugated goat anti-rabbit or mouse IgG was used as a secondary antibody. Bound fragments were detected with ECL chemiluminescence kit (Pierce) and exposed on X-film.

Statistical analysis. The Student's t-test was applied to analyze statistical differences between two groups. $\mathrm{p}<0.05$ was considered to be statistically significant. Each test data from independent experiments were repeated at least 3 times.

\section{Results}

Isolation and culture of CAFs and NFs. To investigate the effect of CAFs on the drug resistance of lung cancer, we isolated CAFs and NFs from lung cancer and normal lung tissues of the same patient following written content. Since CAFs and NFs display similar spindle-like morphology in culture, we carried out immunofluorescence staining and quantitative PCR to compare the expression level of $\alpha$-SMA and FAP. CAFs exhibited a higher amount of $\alpha$-SMA at the mRNA (Fig. 1B) and protein level (Fig. 1A) than NFs, whereas there were no significant differences in FAP expression at the mRNA (Fig. 1B) and protein level (Fig. 1A) between CAFs and NFs. Meanwhile, we also compared the expression and secretion of SDF-1 $\alpha$ from CAF and NFs. Data in Fig. 2A showed that CAFs transcribed much more SDF-1 mRNA than NFs. Furthermore, ELISA data indicated that the concentration of SDF-1 $\alpha$ in the CAF culture supernatant was $9.53 \mathrm{ng} / \mathrm{ml}$, while that in the $\mathrm{NF}$ culture supernatant was only $3.23 \mathrm{ng} / \mathrm{ml}$ (Fig. 2B). Local NFs are considered to be one origin of CAFs. The expression of $\alpha$-SMA and SDF- $1 \alpha$ is significantly upregulated during transformation from NFs to activated CAFs (15). Taken together, these results imply that CAFs derived from patients with lung cancer may be reprogrammed from local NFs and exert their functions via secretion of SDF-1 $\alpha$. 
A
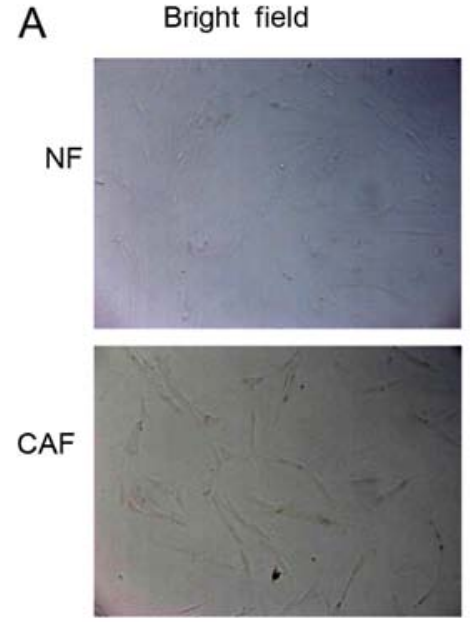

a-sma
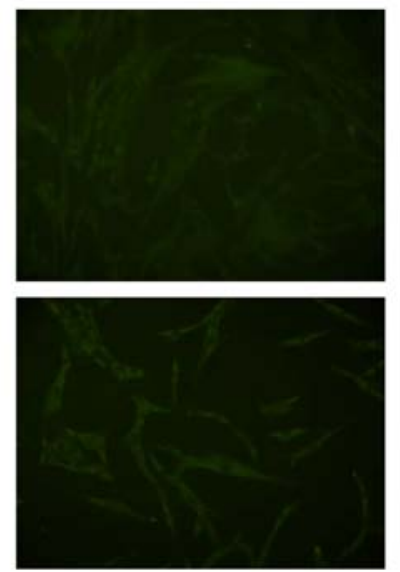

B

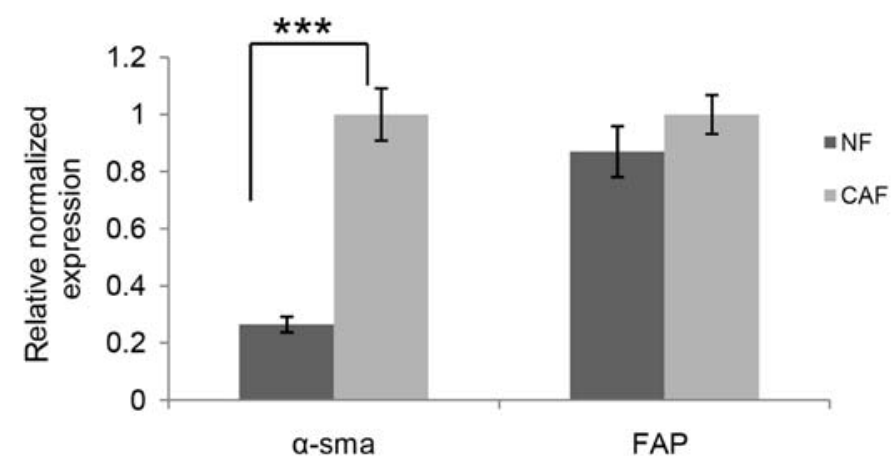

Figure 1. Characterization of NFs and CAFs by immunofluorescence staining and quantitative PCR. (A) CAFs and NFs were both labeled with primary antibodies to $\alpha$-SMA (mouse) and FAP (rabbit). Secondary antibodies to mouse IgG (FITC-labeled) and rabbit IgG (TRITC-labeled) were added afterwards. Images were captured using an inverted fluorescence microscope at a magnification of x200. CAFs had higher $\alpha$-SMA expression than NFs, while they both highly expressed FAP. (B) Quantitative PCR data indicated that CAFs expressed more $\alpha$-SMA instead of FAP than NFs at the mRNA level (n=3; $\left.{ }^{* * *} \mathrm{p}<0.05\right)$.

A

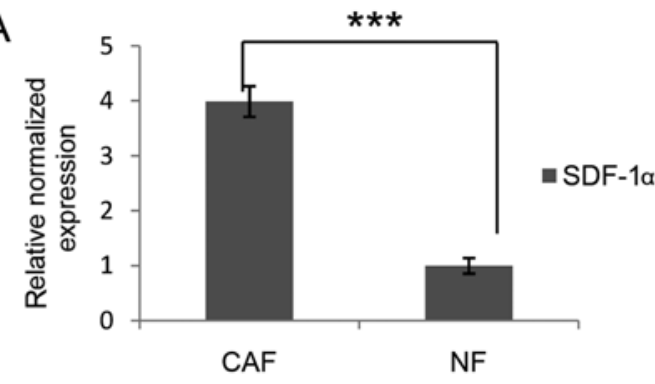

B

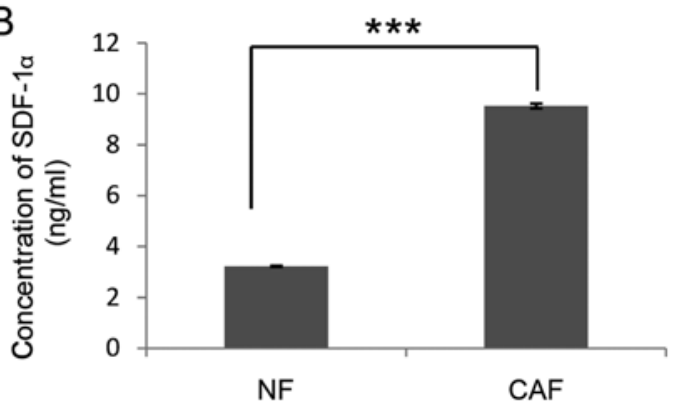

C

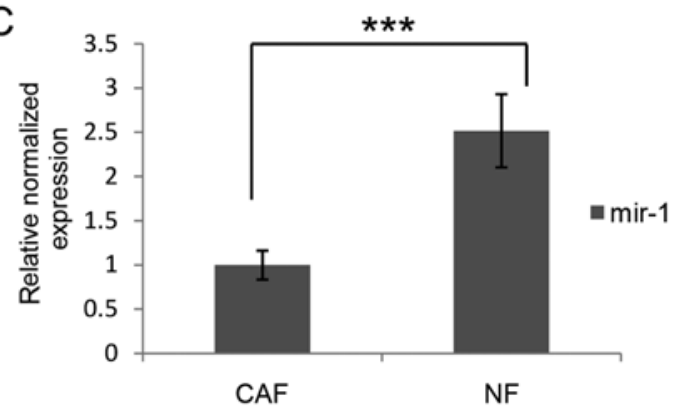

Figure 2. CAFs synthesized and secreted a higher level of SDF-1 $\alpha$ than NFs while NFs expressed more mir-1 than CAFs. (A) Quantative PCR data showed that CAFs expressed a higher amount of SDF-1 $\alpha$ at the mRNA level than NFs $\left(\mathrm{n}=3 ;{ }^{* * *} \mathrm{p}<0.05\right)$. (B) ELISA detected that CAFs secreted more SDF-1 $\alpha$ into the medium than NFs $\left(n=3 ;{ }^{* * *} \mathrm{p}<0.05\right)$. (C) Quantative PCR data showed that CAFs expressed a lower amount of mir-1 at the mRNA level than NFs $\left(\mathrm{n}=3 ;{ }^{* * *} \mathrm{p}<0.05\right)$.
Effects of CXCR4 on cell proliferation and drug resistance of A549 and 95D cells. To illuminate the function of CXCR4 in the cell proliferation and drug resistance of A549 and 95D cells, we performed CXCR4 plasmid transfection and CXCR4 siRNA transfection to upregulate and downregulate its expression level in A549 and 95D cells, respectively. As shown in Fig. 3A and B, CXCR4 upregulation elevated the cell proliferation rate of the A549 and 95D cells, whereas CXCR4 silencing had exactly the opposite effects. Simultaneously, chemoresistance of A549 and 95D to cisplatin (CDDP) was 
A
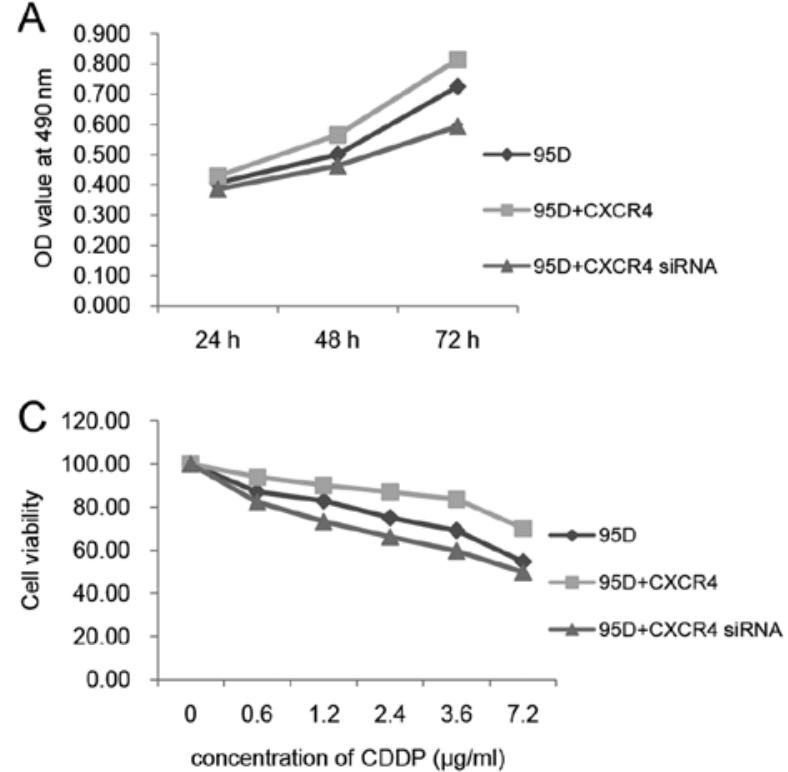

E

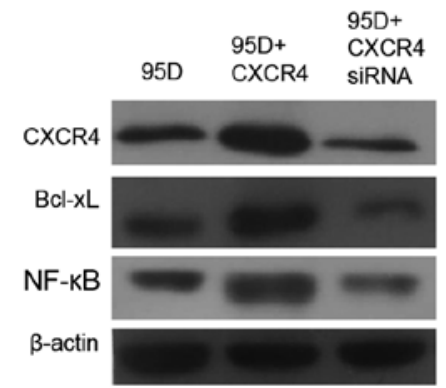

B
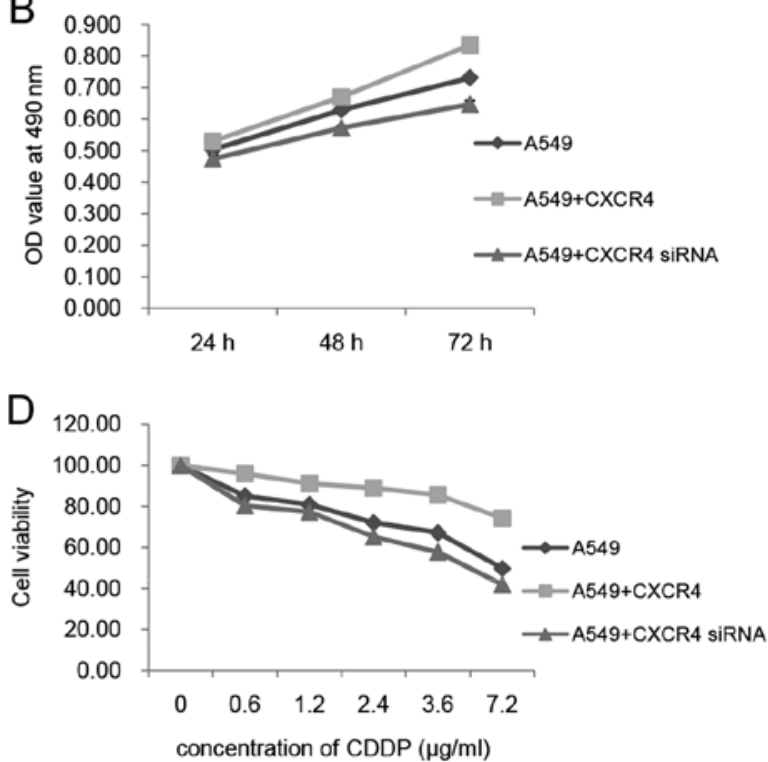

F

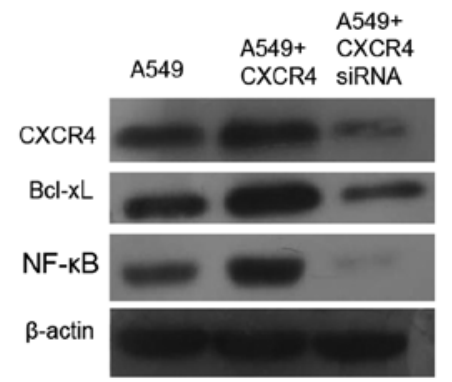

Figure 3. Effects of CXCR4 overexpression or silencing on cell proliferation and drugresistance of A549 and 95D cells. (A and B) MTT assay showed that CXCR4 overexpression facilitated the cell proliferation of A549 and 95D cells and that CXCR4 silencing had the opposite effects. (C and D) MTT assay data indicated that CXCR4 overexpression improved the drug resistance of A549 and 95D cells and that CXCR4 silencing had the opposite effects. Concentrations of cisplatin applied in this assay were $0,0.6,1.2,2.4,3.6$ and $7.2 \mu \mathrm{g} / \mathrm{ml}$. This assay was performed $24 \mathrm{~h}$ after drug addition into cell culture. Cell viability at the concentration of $0 \mu \mathrm{g} / \mathrm{ml}$ was set as $100 \%$; cell viability at all other concentrations was accordingly calculated. (E and F) Western blot assay was applied to assess the protein levels of Bcl-xL and NF- $\kappa \mathrm{B}$ in $95 \mathrm{D}$ and $\mathrm{A} 549$ cells after CXCR4 overexpression or silencing. Expression of Bcl-xL and NF- $\mathrm{B}$ was increased or decreased in the A549 and 95D cells after CXCR4 upregulation or silencing, respectively. Cell samples were collected $24 \mathrm{~h}$ after CXCR4 plasmid or siRNA transfection and lysed with cell lysis buffer after collection. Collected protein samples were stored at $-80^{\circ} \mathrm{C}$. Western blotting was conducted on the second day.

enhanced or suppressed as the CXCR4 expression level was increased or decreased, respectively (Fig. 3C and D). As reported, CXCR4 plays a role in tumor cell growth, survival and drug resistance mainly through the PI3K/AKT/NF- $\mathrm{BB}$ axis as well as the ERK1/2/NF- $\mathrm{B}$ axis (8). Meanwhile, $\mathrm{NF}-\kappa \mathrm{B}$ activation or upregulation usually leads to transcriptional activation of genes such as Bcl-xL to suppress apoptosis (9). Therefore, western blotting was utilized to reveal the involvement of Bcl-xL and $\mathrm{NF}-\kappa \mathrm{B}$ during $\mathrm{CXCR} 4$ overexpression and silencing. As demonstrated in Fig. 3E and F, we obtained similar data from the A549 and 95D cells. The protein levels of Bcl-xL and $\mathrm{NF}-\kappa \mathrm{B}$ were both escalated as CXCR4 was overexpressed, while their protein levels were both declined as CXCR4 was silenced. These data indicated that, in the A549 and 95D cells, activation of NF- $\mathrm{BB}$ and Bcl-xL could be possible signaling transduction factors for CXCR4 to promote its role in cell proliferation and drug resistance.

Effects of CAFs on cell proliferation and drug resistance of A549 and 95D cells. To reveal the effects of CAFs on cell proliferation and drug resistance of A549 and 95D, a
Transwell-base co-culture system and CAF culture supernatant were applied to the A549 and 95D cells, respectively. As in the Transwell-base co-culture system, A549 and 95D cells were seeded in 96-well plates at the concentration of 5,000/ well, and CAFs were seeded in the inserts at the concentration of 5,000/well, as well. As shown in Fig. 4A and B, the OD values of the 95D and A549 cells were both increased after co-culture with the CAFs. Moreover, cell viability of the 95D and A549 cells in the cisplatin suspension was also increased after co-culture with the CAFs. CAFs are routinely cultivated in T25 flasks. When they reached confluency, which was $\sim 1 \times 10^{6}$ in each flask according to our counting, the culture medium was replaced with $5 \mathrm{ml}$ fresh medium. Twenty-four hours later, the culture supernatant was collected. With the addition of the CAF culture supernatant, the OD values of the 95D and A549 cells were also increased compared with that in the control group (Fig. 4A and B). Meanwhile, the cell viability of the 95D and A549 cells in cisplatin suspension was increased with the addition of the CAF culture supernatant as well (Fig. 4C and D). The data we obtained using co-culture techniques and supernatant were consistent with each other. 

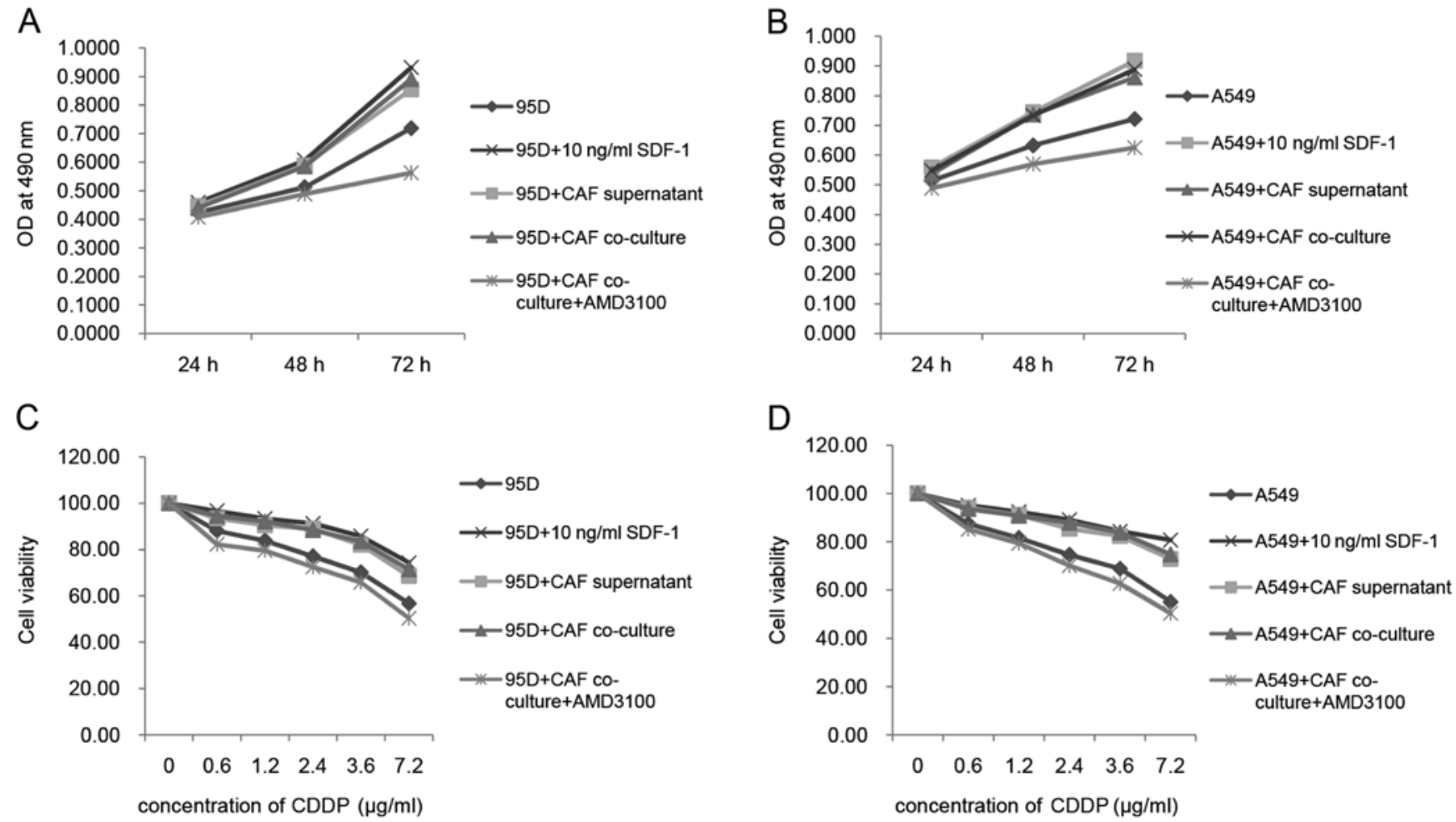

Figure 4. CAFs enhance the cell proliferation and drug resistance of A549 and 95D cells through SDF-1 secretion. (A and B) MTT assay was applied to measure the proliferation rate of A549 and 95D cells after co-cultured with CAFs or addition of CAF supernatant. In the present study, cells + $10 \mathrm{ng} / \mathrm{ml}$ SDF-1 was set as a positive control. CAF supernatant, CAF co-culture and $10 \mathrm{ng} / \mathrm{ml}$ SDF-1 exerted similar positive effects on the proliferation of A549 and 95D cells. AMD3100, one type of CXCR4 inhibitor, was added to the cells co-cultured with CAFs to block the interaction between SDF-1 and CXCR4. In this group, proliferation of the A549 and 95D cells was decreased compared with that in all other groups, even in the control group. (C and D) MTT assay data indicated that drug-resistance of the A549 and 95D cells was increased after co-cultured with CAFs or addition of CAFs supernatant. Concentrations of cisplatin applied in the present study were $0,0.6,1.2,2.4,3.6$ and $7.2 \mu \mathrm{g} / \mathrm{ml}$. This assay was performed $24 \mathrm{~h}$ after drug addition into cell culture. Cell viability at the concentration of $0 \mu \mathrm{g} / \mathrm{ml}$ was set as $100 \%$; cell viability at all other concentrations was accordingly calculated. In the present study, cells $+10 \mathrm{ng} / \mathrm{ml}$ SDF-1 was set as a positive control. CAF supernatant, CAF co-culture and $10 \mathrm{ng} / \mathrm{ml}$ SDF-1 exerted similar positive effects on the drug-resistance of A549 and 95D cells AMD3100 $(10 \mu \mathrm{g} / \mathrm{ml})$, one type of CXCR4 inhibitor, was added to the cells co-cultured with CAFs to block the interaction between SDF-1 and CXCR4. In this group, the drug resistance of the A549 and 95D cells was inhibited compared with that in all other groups, even in the control group.

As reported, CAFs are capable of affecting tumor growth, survival and chemoresistance by secretion of TGF- $\beta 1$, SDF-1 and other small molecules (5). To verify the involvement of SDF-1 in the present study, we simultaneously assessed the effects of SDF-1 $\alpha$ on the cell proliferation and drug resistance of the A549 and 95D cells. As indicated by the data in Fig. 4, SDF-1 $\alpha$ exhibited capability similar to the CAF culture supernatant as well as the CAF co-culture system. Furthermore, we added CXCR4 inhibitor-AMD3100 to test whether or not CXCR4 was involved. As hypothesized, $10 \mu \mathrm{g} / \mathrm{ml}$ AMD3100 attenuated the cell viability of the A549 and 95D cells in the cisplatin suspension and slowed down the cell proliferation rate of the A549 and 95D cells compared with all other groups. These data indicate that the SDF-1/CXCR4 axis played an important role in the effects of CAFs on the cell proliferation and drug resistance of the A549 and 95D cells.

Effects of CAFs on signaling transductions in the A549 and $95 \mathrm{D}$ cells. To elucidate the signaling passways in A549 and $95 \mathrm{D}$ cells that are influenced by the paracrine effect of CAFs, we compared the protein levels of $\mathrm{NF}-\kappa \mathrm{B}$ and $\mathrm{Bcl}-\mathrm{xL}$ among the groups by western blotting. As shown in Fig. 5, the CAF supernatant and CAF co-culture system had similar effects on the expression of CXCR4, NF- $\kappa \mathrm{B}$ and $\mathrm{Bcl}-\mathrm{xL}$. The protein level of CXCR4 was increased by addition of the CAF supernatant as well as co-culture with CAFs. In addition, subsequently, the expression levels of $\mathrm{NF}-\kappa \mathrm{B}$ and $\mathrm{Bcl}-\mathrm{xL}$ were both upregulated. To confirm the participation of SDF-1, we also measured the expression level of $\mathrm{CXCR} 4, \mathrm{NF}-\kappa \mathrm{B}$ and $\mathrm{Bcl}-\mathrm{xL}$ after addition of recombinant human SDF-1. As indicated in Fig. 5A and B, the protein levels of $\mathrm{CXCR} 4, \mathrm{NF}-\kappa \mathrm{B}$ and $\mathrm{Bcl}-\mathrm{XL}$ were all elevated by direct addition of $10 \mathrm{ng} / \mathrm{ml} \mathrm{SDF-1}$ into the cell culture of A549 and 95D. Furthermore, we also assessed the expression of $\mathrm{Bcl}-\mathrm{xL}$ and $\mathrm{NF}-\kappa \mathrm{B}$ after blocking the activation of CXCR4 with AMD3100. As shown in Fig. 5C and D, both Bcl-xL and NF- $\kappa \mathrm{B}$ were downregulated following the addition of $10 \mu \mathrm{g} / \mathrm{ml}$ AMD3100 into the cell culture of A549 and 95D. Taken together, these above data suggested that upregulation of NF- $\mathrm{BB}$ and Bcl-xL through the SDF-1/CXCR4 axis could be the molecular mechanisms behind the paracrine effects of CAFs on the A549 and 95D cells.

Regulation of mir-1 on SDF-1 synthesis in CAFs and its subsequent effects on downstream signaling transduction in A549 and 95D cells. mir-1, as a tumor-suppressor microRNA, was reported to be expressed at a very low level in lung cancer and other cancer types (12). SDF-1 was identified as one of its targets in head and neck tumors (13). In the present study, 

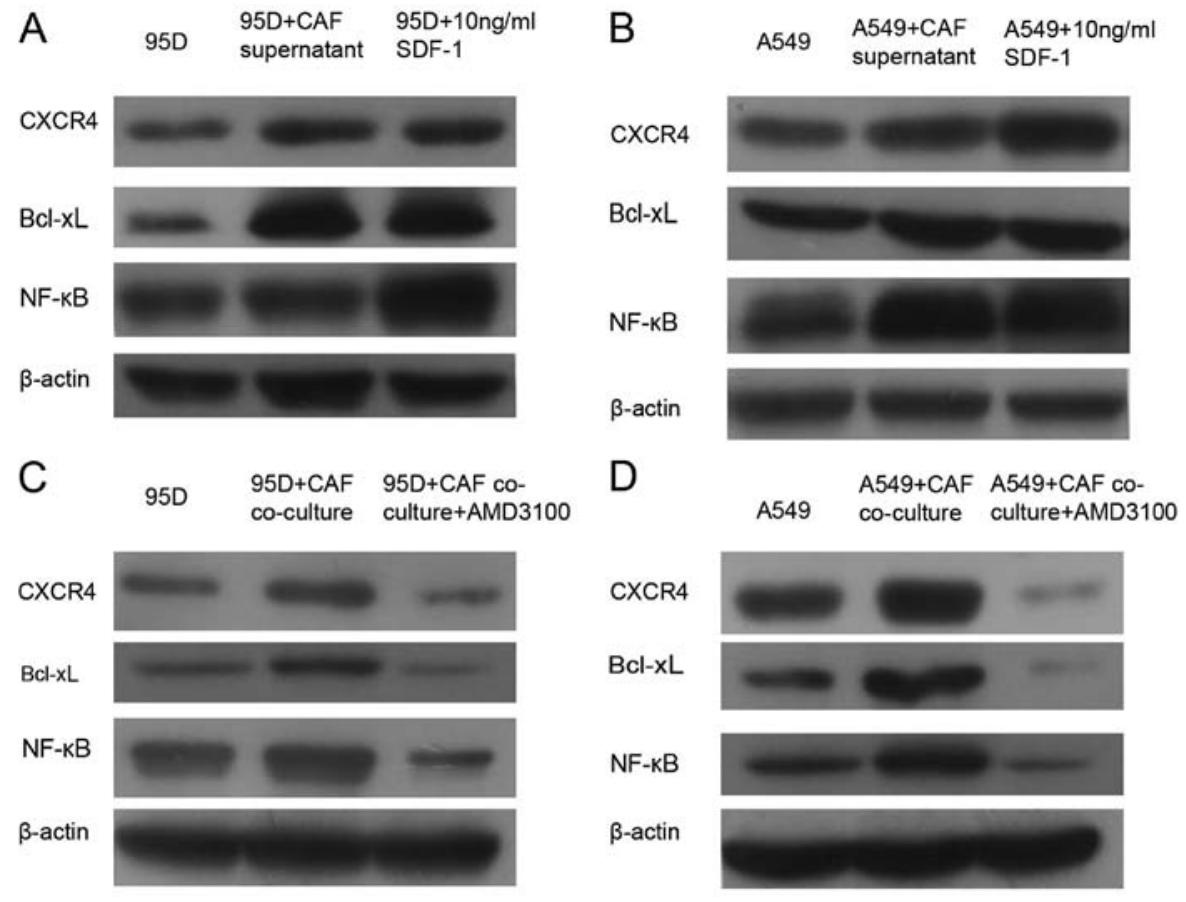

Figure 5. CAFs increase the protein levels of Bcl-xL and NF- $\mathrm{KB}$ in the A549 and 95D cells. (A-D) Western blot assay was applied to assess the protein levels of Bcl-xL and NF- $\mathrm{KB}$ in the 95D and A549 cells after co-culture with CAFs or addition of the CAF supernatant. In the present study, cells + $10 \mathrm{ng} / \mathrm{ml}$ SDF-1 was set as a positive control. CAF supernatant, CAF co-culture and $10 \mathrm{ng} / \mathrm{ml}$ SDF-1 exerted similar positive effects on the expression of Bcl-xL and NF- $\mathrm{kB}$ in the A549 and 95D cells. AMD3100 (10 $\mu \mathrm{g} / \mathrm{ml})$, one type of CXCR4 inhibitor, was added to the cells co-cultured with CAFs to block the interaction between SDF-1 and CXCR4. In this group, the protein levels of Bcl-xL and NF-kB in the A549 and 95D cells were decreased compared with that in all other groups, even in the control group. Cell samples were collected $24 \mathrm{~h}$ after all treatments and lysed with cell lysis buffer right after collection. Collected protein samples were stored at $-80^{\circ} \mathrm{C}$. Western blotting was conducted on the second day.

A

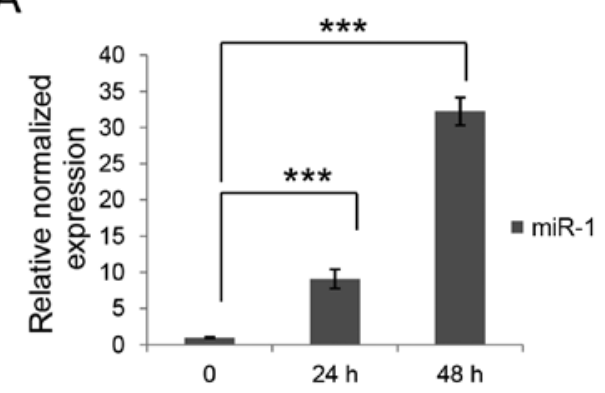

C

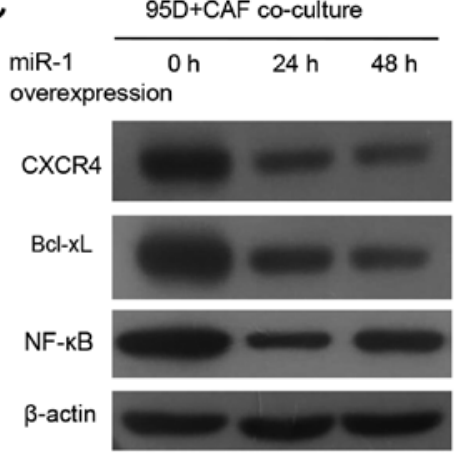

B

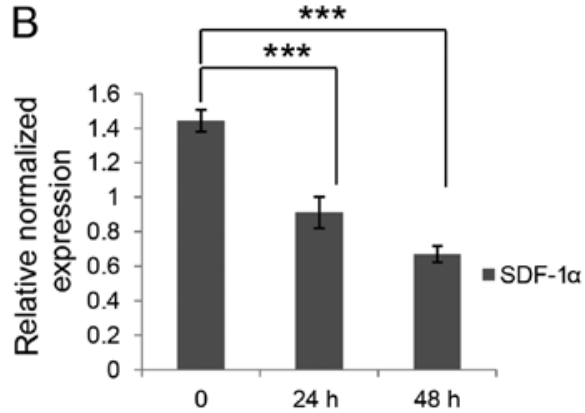

D
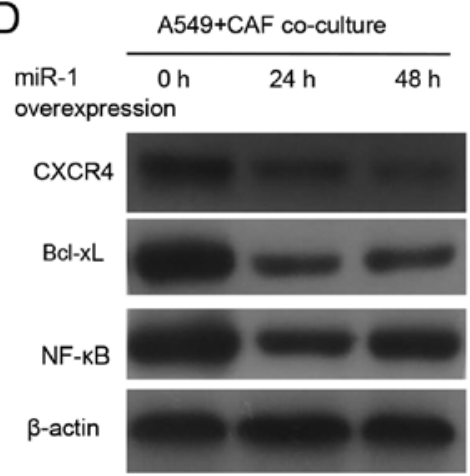

Figure 6. MicroRNA mir-1 negatively regulates the expression of SDF-1 $\alpha$ in CAFs and consecutively downregulates the protein levels of CXCR4, Bcl-xL and NF- $\mathrm{kB}$ in A549 and 95D cells. (A) Quantative PCR was conducted to evaluate transfection efficiency of mir-1 mimics into CAFs. The quantity of mir-1 was significantly increased 24 and $48 \mathrm{~h}$ after transfection (9.1 and 32.3 vs. $1 ;{ }^{* * *} \mathrm{p}<0.05$ ). (B) The mRNA level changes of SDF-1 $\alpha$ in CAFs were measured by quantitative PCR 24 and $48 \mathrm{~h}$ after mir-1 transfection. In the present study, the quantity of SDF-1 $\alpha$ in the untransfected group (named as 0 ) was set as 1 . The quantity of SDF-1 $\alpha$ in CAFs 24 and $48 \mathrm{~h}$ after transfection was $0.63\left({ }^{* * *} \mathrm{p}<0.05\right)$ and $0.46\left({ }^{* * *} \mathrm{p}<0.05\right)$, respectively. (C and D) Western blotting was conducted to detect protein level changes of CXCR4, Bcl-xL and NF- $\mathrm{KB}$ in the A549 and 95D cells after co-cultured with the mir-1-transfected CAFs. Protein levels of all of these 3 factors were decreased in the A549 and 95D cells after co-culture with the mir-1-transfected CAFs. Cell samples were collected $24 \mathrm{~h}$ after co-culture and lysed with cell lysis buffer after collection. Collected protein samples were stored at $-80^{\circ} \mathrm{C}$. Western blotting was conducted on the second day. 
we upregulated mir-1 expression in the CAFs by mir-1 mimic transfection (Fig. 6A). In addition, consequently, the expression level of SDF-1 was decreased as indicated in Fig. 6B. We also detected the expression levels of CXCR4, NF- $\kappa \mathrm{B}$ and Bcl-xL in the A549 and 95D cells after co-culture with mir-1-overexpressing CAFs. As demonstrated by the data in Fig. $6 \mathrm{C}$ and D, the protein levels of CXCR4, NF- $\kappa \mathrm{B}$ and Bcl-xL in the A549 and 95D cells declined after co-culture with the mir-1-overexpressing CAFs, although changes were not apparently time-dependent. These results revealed that mir-1 negatively regulated the expression of SDF-1 and that mir-1 overexpression consequently downregulated the expression of CXCR4, NF- $\kappa \mathrm{B}$ and Bcl-xL in the A549 and 95D cells by co-culturing with mir-1-upregulated CAFs.

\section{Discussion}

The tumor microenvironment plays an important role in cancer development and progression. CAFs, the dominant component of the tumor microenvironment, have been shown to be crucial for tumor cell proliferation, survival and therapeutic resistance (4). In this present study, we first isolated CAFs from patient tissues and demonstrated that they promoted cell proliferation and chemoresistance to cisplatin in lung cancer cell lines A549 and 95D in a paracrine manner. Secondly, using ELISA and quantitative PCR, we found that a higher amount of SDF-1 existed in the CAFs when compared with that in the NFs. Thirdly, we found that SDF-1 facilitated lung cancer cell proliferation and drug resistance via the CXCR4-mediated signaling passway which involved NF- $\kappa \mathrm{B}$ and Bcl-xL. Moreover, we also confirmed that the expression level of SDF-1 in CAFs was negatively regulated by microRNA mir-1.

CAFs are heterogeneous and poorly defined to date. $\alpha-S M A$, FAP, vimentin and many other markers have been reported to characterize them (16). In the present study, we employed $\alpha$-SMA and FAP. As shown in our results, $\alpha$-SMA and FAP were both highly expressed in the CAFs, which indicated that lung cancer CAFs may resemble myofibroblasts since co-expression of $\alpha$-SMA and FAP is characteristic of myofibroblasts (4). However, we also found that FAP was expressed in NFs and no significant difference at the mRNA level was detected between NFs and CAFs. Meanwhile, a higher expression level of SDF-1 was observed in the CAFs when compared with that in the NFs. Therefore, $\alpha$-SMA and SDF-1 may be superior markers of lung cancer CAFs than FAP.

Therapeutic resistance of lung cancer, particularly NSCLC, is mainly due to improved survival ability of cells and metastasis (1). Numerous studies have established the correlation between CXCR4 expression and poor prognosis in NSCLC. CXCR4 overexpression was reported to be associated with poor survival in stage IV NSCLC patients (17). In our results, elevated CXCR4 expression in lung cancer cell lines A549 and 95D promoted cell proliferation and drug resistance to cisplatin. The protein levels of $\mathrm{NF}-\kappa \mathrm{B}$ and $\mathrm{Bcl}-\mathrm{xL}$ were found to be increased in the CXCR4-overexpressing A549 and 95D cells as well as in tumor cells co-cultured with CAFs. Furthermore, co-culture with the CAFs also induced upregulation of CXCR4 expression in the A549 and 95D cells, which was attenuated by addition of AMD3100. In previous studies by other research groups, NF- $\mathrm{B}$ was found to suppress apoptosis by activating TRAF1 and TRAF2, as well as bcl-2 homologues A1/Bfl-1 and Bcl-xL (9). Moreover, NF- $\kappa$ B was also found to be involved in cell growth and angiogenesis by regulating the expression of ICAM-1 and Cox-2 (9). Taken together, our data may provide explanations for the correlation between CXCR4 expression and the poor outcome of lung cancer and offer evidence for the potential therapeutic application of targeting CAFs or the SDF-1/CXCR4 axis.

MicroRNAs exert their regulatory role in a variety of biological process. Recently, they have been demonstrated to function as oncogenes or tumor-suppressor genes in many types of cancers. mir-1 as well as mir-148 have been identified as tumor suppressors $(10,11,18)$. Both were discovered to be expressed at a very low level in many types of tumors including lung cancer. mir-148 was reported to suppress metastasis and mir-1 was found to be involved in tumor cell proliferation, metastasis and apoptosis $(10,18)$. However, not much is known concerning their functions in CAFs. In the present study, mir-1 expression in lung cancer CAFs was measured, which was lower than that in the NFs. Our data also indicated that mir-1 negatively regulates the expression of SDF-1, which plays an important role during the interaction between CAFs and tumor cells. Thus, microRNA expression was altered in CAF formation and affected CAF functions. Meanwhile, to the best of our knowledge, this was the first study to report the function of mir-1 in lung cancer CAFs. In the future, more research is warranted to obtain a better understanding of the functions of mirRNAs in CAFs, in particular in the whole tumor microenvironment.

In summary, we discovered that CAFs were capable of influencing the cell proliferation and drug resistance of A549 and 95D cells by SDF-1 secretion. In addition, SDF-1 upregulated the expression of CXCR4 which subsequently elevated the expression of NF- $\mathrm{BB}$ and Bcl-xL. Meanwhile, microRNA mir-1 mediated the expression of SDF-1 in the CAFs. Conclusively, we revealed a mir-1/SDF-1/CXCR4/ $\mathrm{NF}-\kappa \mathrm{B} / \mathrm{Bcl}-\mathrm{xL}$ signaling pathway behind the interaction between $\mathrm{CAFs}$ and the lung cancer cell lines, which explains the reason why CAFs increased the proliferation rate and drug resistance capacities of the A549 and 95D cells. These results also identify CAFs as a potential therapeutic target in tumor treatment.

\section{Acknowledgements}

The present study was supported by the National Natural Science Foundation of China (no. 81370128).

\section{References}

1. Wald O, Shapira OM and Izhar U: CXCR4/CXCL12 axis in non small cell lung cancer (NSCLC) pathologic roles and therapeutic potential. Theranostics 3: 26-33, 2013.

2. Siegel R, Naishadham D and Jemal A: Cancer statistics, 2012. CA Cancer J Clin 62: 10-29, 2012.

3. Kim SH, Choe C, Shin YS, Jeon MJ, Choi SJ, Lee J, Bae GY, Cha HJ and Kim J: Human lung cancer-associated fibroblasts enhance motility of non-small cell lung cancer cells in co-culture. Anticancer Res 33: 2001-2009, 2013.

4. Franco OE, Shaw AK, Strand DW and Hayward SW: Cancer associated fibroblasts in cancer pathogenesis. Semin Cell Dev Biol 21: 33-39, 2010 
5. Karagiannis GS, Poutahidis T, Erdman SE, Kirsch R, Riddell RH and Diamandis EP: Cancer-associated fibroblasts drive the progression of metastasis through both paracrine and mechanical pressure on cancer tissue. Mol Cancer Res 10: 1403-1418, 2012.

6. Chatterjee S, Behnam Azad B and Nimmagadda S: The intricate role of CXCR4 in cancer. Adv Cancer Res 124: 31-82, 2014.

7. Chen Y, Jacamo R, Konopleva M, Garzon R, Croce C and Andreeff M: CXCR4 downregulation of let-7a drives chemoresistance in acute myeloid leukemia. J Clin Invest 123: 2395-2407, 2013.

8. Teicher BA and Fricker SP: CXCL12 (SDF-1)/CXCR4 pathway in cancer. Clin Cancer Res 16: 2927-2931, 2010.

9. Perona R and Sánchez-Pérez I: Control of oncogenesis and cancer therapy resistance. Br J Cancer 90: 573-577, 2004.

10. Han C, Yu Z, Duan Z and Kan Q: Role of microRNA-1 in human cancer and its therapeutic potentials. Biomed Res Int 2014 428371, 2014.

11. Aprelikova O and Green JE: MicroRNA regulation in cancerassociated fibroblasts. Cancer Immunol Immunother 61: 231-237, 2012.

12. Li J, Dong X, Wang Z and Wu J: MicroRNA-1 in cardiac diseases and cancers. Korean J Physiol Pharmacol 18: 359-363, 2014.

13. Leone V, D'Angelo D, Rubio I, de Freitas PM, Federico A, Colamaio M, Pallante P, Medeiros-Neto G and Fusco A: MiR-1 is a tumor suppressor in thyroid carcinogenesis targeting CCND2, CXCR4, and SDF-1alpha. J Clin Endocrinol Metab 96 E1388-E1398, 2011.
14. van Solingen C, de Boer HC, Bijkerk R, Monge M, van OeverenRietdijk AM, Seghers L, de Vries MR, van der Veer EP, Quax PH, Rabelink TJ, et al: MicroRNA-126 modulates endothelial SDF-1 expression and mobilization of $\mathrm{Sca}-1^{+} / \mathrm{Lin}$ progenitor cells in ischaemia. Cardiovasc Res 92: 449-455, 2011.

15. Huang L, Xu AM, Liu S, Liu W and Li TJ: Cancer-associated fibroblasts in digestive tumors. World J Gastroenterol 20: 17804-17818, 2014

16. Du H, Chen D, Zhou Y, Han Z and Che G: Fibroblast phenotypes in different lung diseases. J Cardiothorac Surg 9: 147, 2014.

17. Otsuka S, Klimowicz AC, Kopciuk K, Petrillo SK, Konno M, Hao D, Muzik H, Stolte E, Boland W, Morris D, et al: CXCR4 overexpression is associated with poor outcome in females diagnosed with stage IV non-small cell lung cancer. J Thorac Oncol 6: 1169-1178, 2011.

18. Hanoun N, Delpu Y, Suriawinata AA, Bournet B, Bureau C, Selves J, Tsongalis GJ, Dufresne M, Buscail L, Cordelier P, et al: The silencing of microRNA 148a production by DNA hypermethylation is an early event in pancreatic carcinogenesis. Clin Chem 56: 1107-1118, 2010. 